\title{
Monitoring of abrasive water jet (AWJ) cutting using sound detection
}

Received: 19 November 2002 / Accepted: 19 February 2003 / Published online: 12 May 2004

(C) Springer-Verlag London Limited 2004

\begin{abstract}
Our main objective in the present work is to develop a methodology and create a system for the abrasive water jet (AWJ) machining process control. In the case of AWJ cutting, besides the cutting head traverse rate, the distance between the mixing tube and the workpiece, designated as the stand-off distance, has a predominant influence on the workpiece quality. The control of the traverse rate is performed by the machine controller. The stand off-distance control during the machining represents a problem because no effective on-line in real-time stand-off distance detection system has been developed yet. The detection of the stand-off distance during cutting enables better AWJ machining process control. order to monitor the standoff distance, we measure the emitted sound generated during the AWJ straight cut operation and analyse its characteristic attributes. In order to verify the proposed stand-off distance monitoring methods, a set of experiments was carried out. The signal analysis was performed in both time and frequency domain. The obtained results show an evident influence of the stand-off distance on sound emission. Thus, efficient control of the AWJ cutting process through sound detection appears to be viable.
\end{abstract}

B. Jurisevic (

Swiss Competence Centre of Water Jet Technology,

University of Applied Sciences,

Aargau, Klosterzelgstrasse, CH-5210 Windisch, Switzerland

E-mail: b.jurisevic@fh-aargau.ch

Tel.: +41-56462-4544

Fax: $+41-56462-4542$

D. Brissaud ( $\square)$

Laboratory "Sols Solides Structures",

University of Grenoble,

Domain Universitaire, B.P. no 53, 38041 Grenoble Cedex 9, France

E-mail: daniel.brissaud@hmg.inpg.fr

Tel.: +33-4768-27-006

Fax: +33-4768-27-043

M. Junkar (

Laboratory for Alternative Technologies,

University of Ljubljana,

Askerceva 6, 1000 Ljubljana, Slovenia

E-mail: miha.junkar@fs.uni-lj.si

Tel: +386-1-4771-730

Fax: +386-1-2518-567
Keywords Abrasive water jet - AWJ - Process monitoring Sound detection $\cdot$ Sound generation sources - SGS .

Stand-off distance

\section{Introduction}

The abrasive water jet machining (AWJ) is a nonconventional manufacturing process. The first industrial applications of the process started in the early 1980s. AWJ is mostly used for contour cutting in one plane. Recently, the possibilities of using the process for milling, drilling, etc. have been explored. The major benefit of the AWJ is the capability of machining virtually any material independently of material properties like hardness, structure, composition, physical properties, etc. Comparing with other complementary machining processes, no heat affected zone (HAZ) on the workpiece is produced.

Many complex mechanisms of material removal and a huge quantity of particles involved in the process produce a strongly nonlinear and stochastic behaviour of the system. The generated surface depends on several machining parameters and workpiece properties. Because the AWJ process is a dynamic system, the interactions of the system inputs (machining parameters and workpiece properties) play an important role on process evolution.

From the technological point of view, the most interesting machining parameters are the cutting head traverse rate, the water pressure, the abrasive mass flow rate and the stand-off distance between the mixing tube and the workpiece. All these parameters can be controlled during AWJ machining. Other parameters, like cutting head components' geometry, abrasive properties and workpiece material properties are unchanged during the process.

Between the controllable parameters, the stand-off distance is the more appropriate to control during the process in order to improve the machining quality. The influence of the standoff distance should not be neglected during the AWJ process optimisation. It is known that there exists an optimal value of stand-off distance, which depends on machining conditions [1] This optimum value allows the maximum depth of cut. Using 
the optimal stand-off distance allows higher traverse speed and consequently a machining cost reduction. Other authors [2] give a deeper explanation about the existence of an optimal value of stand-off distance as shown in Fig. 1. Below this optimal value of stand-off distance, the water does not exit from the mixing tube quickly enough. It accumulates in the mixing tube, acting as a shock absorber. It the extreme case, the water blows backward in the mixing tube, causing the abrasive jamming. When the stand-off distance increases over the optimal value, the cutting depth decrease exponentially due to the friction of the jet in the air. Besides on the depth of cut, the influence of the standoff distance is significant on the width of the cut, which increases with a stand-off increase [3]. As well, the burr height increases with stand-off distance increase [4]. Similar increasing trends with stand-off distance increases are present on the width of initial damage zone on the workpiece [3], on the flank angle of the cut [3] and on the taper of the cut ration [5].

Our objective is to control or, in other words, keep the standoff distance at the optimal value during the AWJ cutting process using the generated sound monitoring. The generated sound during the process is a result of many complex mechanisms. Recognition of the absolute value of the stand-off distance is difficult to achieve. More perspective is gained as the detection of the standoff distance changes in reference to the preset value. An adaptive control system (ACS), which is able to keep constant the standoff distance, would be of great importance for the overall process efficiency.

\section{Experiments}

The experiments were performed in the Laboratory for Alternative Technologies (LAT) at the University of Ljubljana. The employed AWJ machine was a commercial system made by the OMAX corporation (type 2,652 A). The water pressure was kept between 2,350 and 2,450 bar and the abrasive was Garnet mesh 80 . The orifice (water nozzle) diameter was $0.381 \mathrm{~mm}$ (0.015 inch), while the mixing tube diameter was $1.016 \mathrm{~mm}$ (0.04 inch) and the length was $101.6 \mathrm{~mm}$ ( 4 inch). The workpiece material was an aluminium alloy $\left(\mathrm{AlMgSi}_{3}\right)$ with hardness $60 \mathrm{HRb}$. For the experiments, two different workpiece thicknesses were taken, namely 6.1 and $50 \mathrm{~mm}$. In order to obtain an equivalent quality of the cut in both cases, we applied two different AWJ cutting head traverse rates. The AWJ cutting head traverse rates were $6.5 \mathrm{~mm} / \mathrm{s}$ for workpiece thickness $6.1 \mathrm{~mm}$ and $0.5 \mathrm{~mm} / \mathrm{s}$ for workpiece thickness $50 \mathrm{~mm}$. In the first stage of the experiment, we pierced the material, then we made a straight cut and finally we exited from the workpiece. Only the part of the signal regarding the straight cut was analysed (Fig. 2).

The data were recorded in a wav file format on the PC (Pentium class) with the acquisition card with $48 \mathrm{kHz}$ sampling frequency and 16-bit resolution. We take approximately 13 seconds of the recorded signal of the straight cut (area 4 on Fig. 2) to have $2^{19}$ points for the further Fast Fourier Transform.

To find the relation between the stand-off distance and the generated Sound, all the machine parameters were kept constant

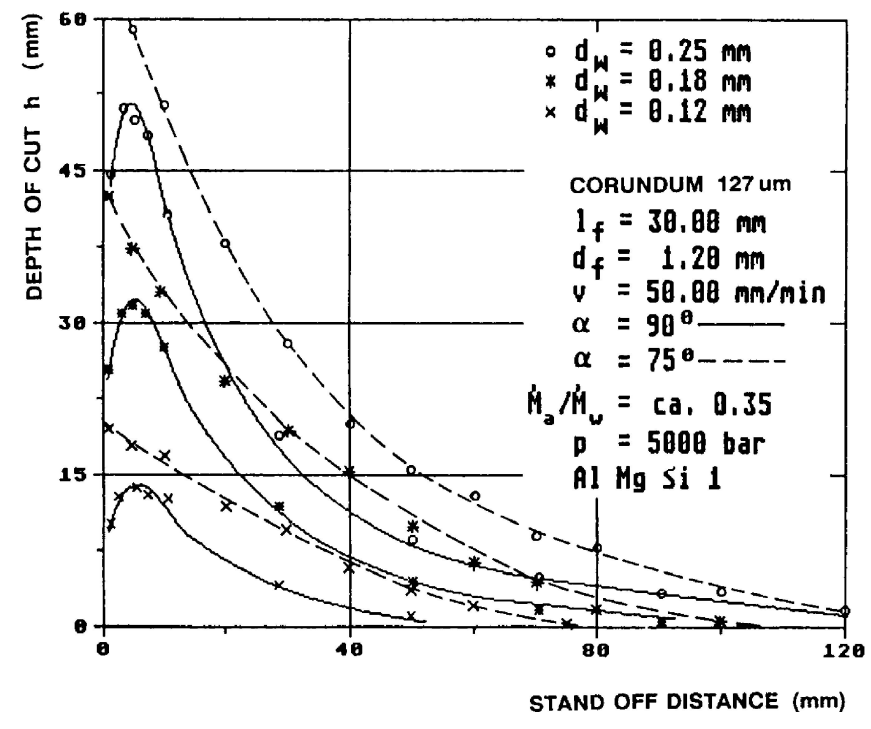

Fig. 1. Depth of cut depending on the stand-off distance and blasting angle [2]

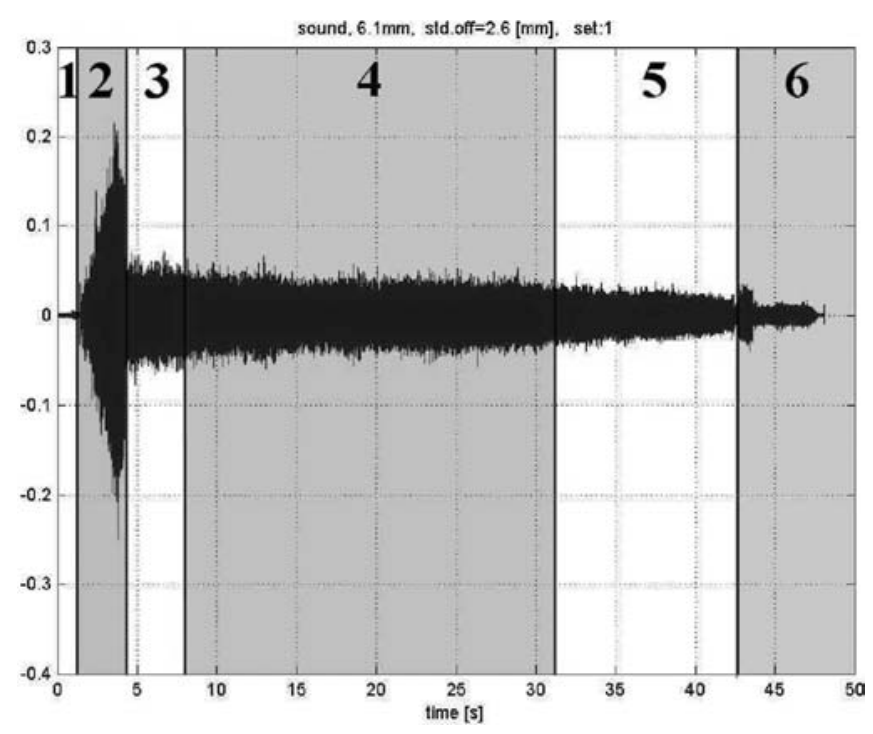

Fig. 2. Recorded signal during all experiment cycles

except the stand-off distance. The cutting head traverse rate was different for each of two workpiece thicknesses in order to generate the same cut quality for both workpiece thicknesses.

At this point, we suppose that, if we machine with the same quality regime, we could generate similar (material removal) mechanisms (in the process) enabling a comparison of the results obtained in both workpiece thickness cases. We applied 11 different stand-off distances, raised between $0.5 \mathrm{~mm}$ and $10 \mathrm{~mm}$ (Table 1). Particular attention was given to the stand-off around $2.5 \mathrm{~mm}$ according to the optimal stand-off distance.

All experiments were repeated three times at the same setting of machine parameters. Thus, we have obtained 33 sets of data for further analyses for each of two workpiece thicknesses. The experiments were designed to enable more tests in future results 
Table 1. Experimental stand-off distances

Stand-off distance [mm]

\begin{tabular}{lllllllllll}
\hline 0.5 & 1 & 1.5 & 2 & 2.3 & 2.6 & 3 & 3.5 & 4 & 5 & 10 \\
\hline
\end{tabular}

analysis. The range of the acquired data was bigger than required for our analysis. For this reason, we installed also a piezzo sensor on the workpiece to measure the acoustic emission, but the results did not reach our expectations. Regarding the measurements with the piezzo sensor, the problems occurs because the workpiece on which we measured the acoustic emission drastically changed its geometry during the experiment and consequently the dynamic condition, which give a different acoustic emission response.

\section{Data evaluation method and definition of attributes}

The recorded data were analysed in both time and frequency domains. Only a part of the signal regarding the straight cut (area 4 on Fig. 2) was analysed. We observe the root mean square (RMS) value in the time domain and the amplitude cumulative sum (ACS) of the signal power spectra in the frequency domain.

The RMS value (Eq. 1) was computed on the part of the signal corresponding to the straight cut. $N$ represents the number of points in the signal and $s s a$ is the amplitude of the sound signal,

$R M S=\sqrt{\frac{1}{N} \cdot \sum_{i=1}^{N} s s a_{i}^{2}}$

To obtain the ACS, we must perform the FFT on the data, but first the signal must be filtered and windowed. Both operations on the

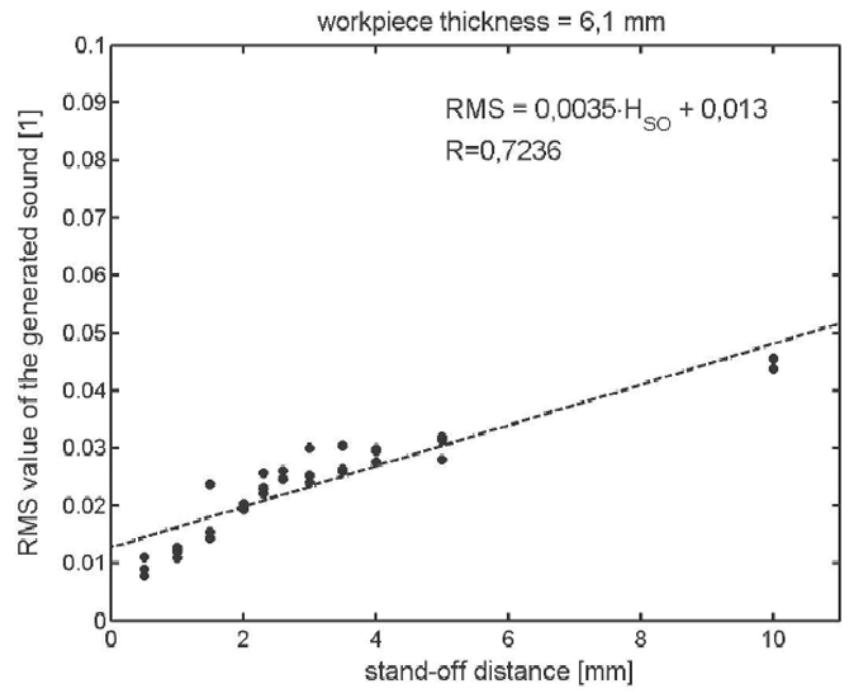

Fig. 3. RMS value for workpiece thickness $6.1 \mathrm{~mm}$ (left) and $50 \mathrm{~mm}$ (right) signal were executed numerically after the experiments. For the signal filtering, we used an elliptic-type pass-band, digital filter with the cut-off frequency up to $15 \mathrm{kHz}$ and over $18 \mathrm{kHz}$. Due to the complexity of the process, the cut-off frequency was defined by a cut-and-try method. The cut-off frequency was defined after several analyses and results as the more appropriate frequency area for stand-off distance characterisation in our case. Other authors $[6,7]$ suggest that a generated sound during the AWJ process is more representative in the frequency area over $20 \mathrm{kHz}$. Because we were sampling the signal with $48 \mathrm{kHz}$, theoretically we couldn't analyse the signal at frequency over $24 \mathrm{kHz}$, according to the Nyquist theorem (which is valid only for periodic signals). But practically, due to the stochastic and nonperiodic nature of the recorded signal, the top edge for the cut-off frequency analysis was set to the lower $18 \mathrm{kHz}$ cut-off frequency. For the signal windowing, we use a Hanning window. After the windowing operation, we subtracted the mean amplitude of the signal from the signal itself (in this way, the new average amplitude of the recorded signal was zero). This prevents the obscuration of the low spectral components by the high-amplitude spectral components.

For the calculation of the ACS at the observed frequency $\omega_{A}$, we have to make a sum of all power spectra amplitudes spa up to the amplitude at the observed frequency (Eq. 2),

$A C S_{\omega A}=\sum_{i=1}^{A} s p a_{\omega i}$.

With this method, we can compare the accumulation of power spectra at frequencies for different stand-off distances in the observed frequency area.

When comparing the analyses in time and frequency domains, it is evident that the first one is much faster for computing and thus enables a real-time monitoring with the state-of-the-art equipment used in the AWJ workshops.

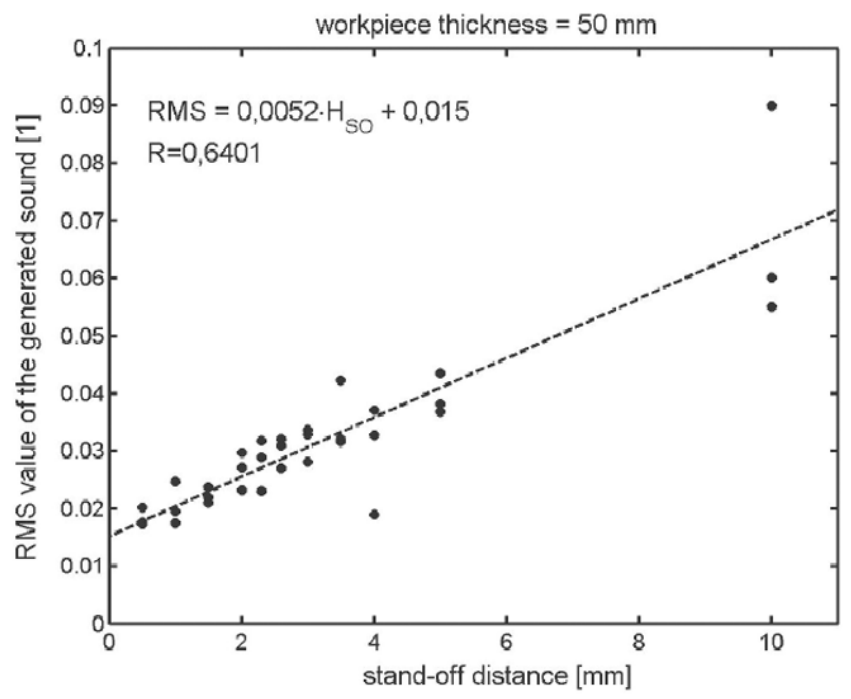




\section{Results}

The observed attributes of the emitted sound (the RMS value of the sound signal in the time domain and the ACS of the signal power spectra in the frequency domain) show distinct a relation with the stand-off distance (statistically confirmed with the analyses of variance). We made the analyses separately for both workpiece thickness $(6.1$ and $50 \mathrm{~mm})$. Better results were obtained in the case of workpiece thickness $6.1 \mathrm{~mm}$.

If we depict the RMS values with the corresponding stand-off distances in a diagram (Fig. 3). We can clearly see that the RMS value increases when the stand-off distance increases. This relation is more obvious for the workpiece thickness $6.1 \mathrm{~mm}$ (Fig. 3, left).

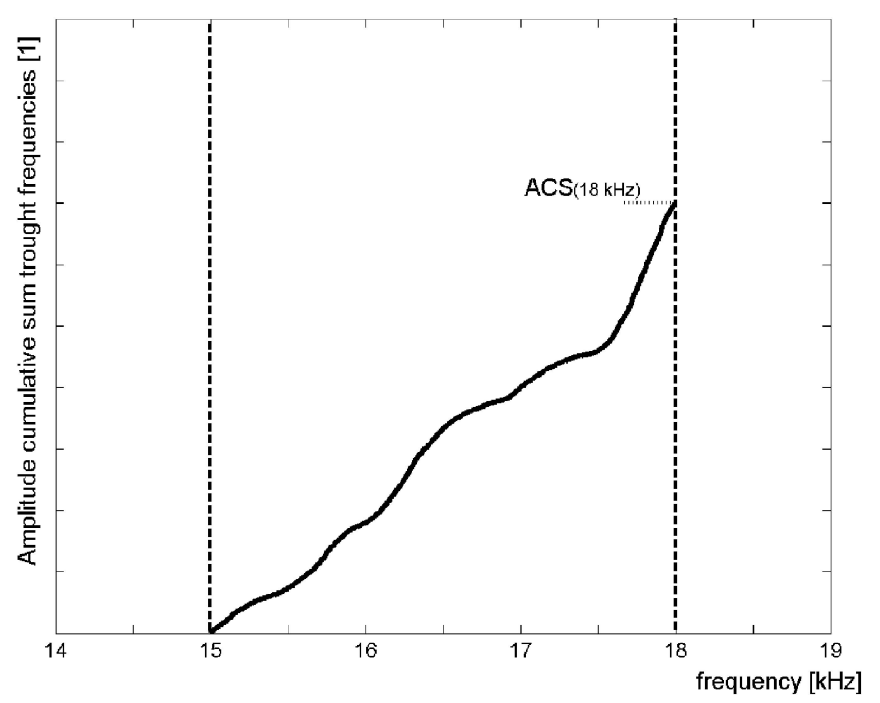

Fig. 4. ACS value at the upper edge of the observed frequency area

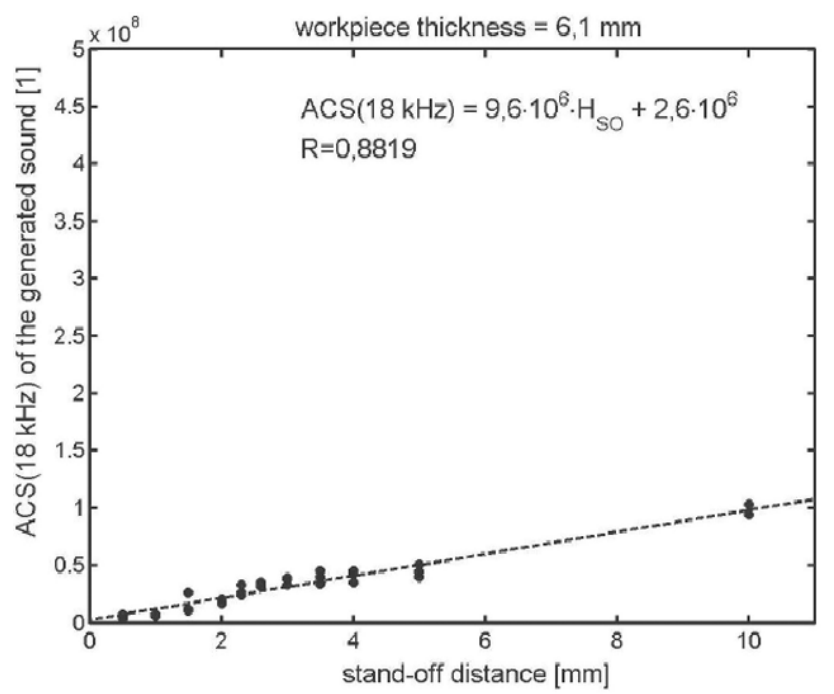

Fig. 5. Mean and max ACS for workpiece thickness $6.1 \mathrm{~mm}$ (left) and $50 \mathrm{~mm}$ (right)
In the frequency domain, we computed the ACS for each stand-off distance. As in the case of the RMS value, the ACS showed a better relation with the stand-off distance for the workpiece thickness $6.1 \mathrm{~mm}$.

To have a better understanding of the resulting ACS, we evaluated the ACS value at the upper edge of the observed frequency area for every single stand-off distance as shown in Fig. 4. These attributes of the ACS are depicted in the diagram to the corresponding stand-off distance (Fig. 5). As expected, the results of the experiments conducted on the workpiece of $6.1 \mathrm{~mm}$ thickness (Fig. 5-left) show better correlation with the stand-off distance compared with those obtained on workpiece thickness $50 \mathrm{~mm}$.

The results are encouraging. Even if we don't perform a large set of Experiments, we can observe the correlation between the sound signal evaluated in both time and frequency domains and the stand-off distance (Fig. 3, Fig. 5).

\section{Discussion}

From the presented studies, we can clearly see that the results are much better in the case of workpiece thickness $6.1 \mathrm{~mm}$, compared with the workpiece thickness $50 \mathrm{~mm}$. An explanation can be found in Fig. 6, where we make a list of possible soundgeneration sources during the AWJ machining process. If we increase the workpiece thickness, we simultaneously increase the sound source number 3 , which represents the cutting zone. In this case, the contribution of the sound source 2 , which depends on the stand-off distance, is smaller and the resulting relation between the sound emission attributes and the stand-off distance is not so clear.

With a better knowledge of the sound generation mechanisms in each of the five sound generation zones that we defined, an empirical model could be made. The description of a simplified form of the sound generation mechanisms in the stand-off

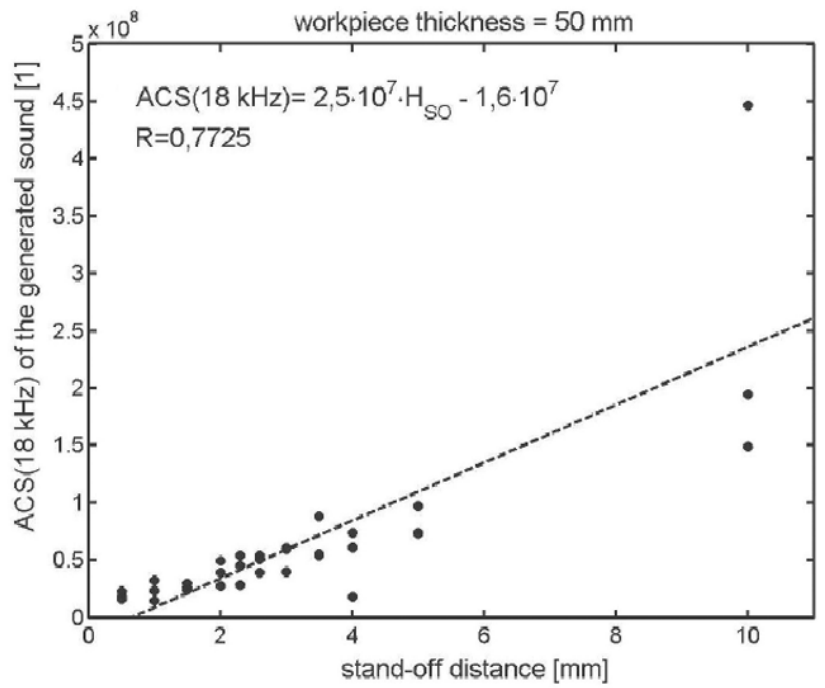


Fig. 6. Proposed sound generation sources

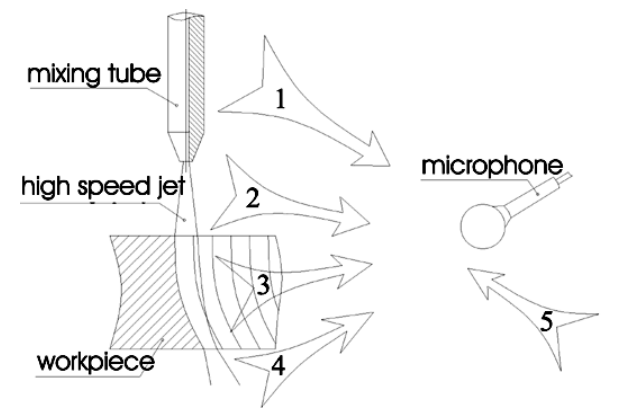

1 ....generated sound from the cutting head

2....generated sound from stand-off area

3 ....generated sound from the cutting zone

4....sound of the jet after cutting

5 ....disturbances environment

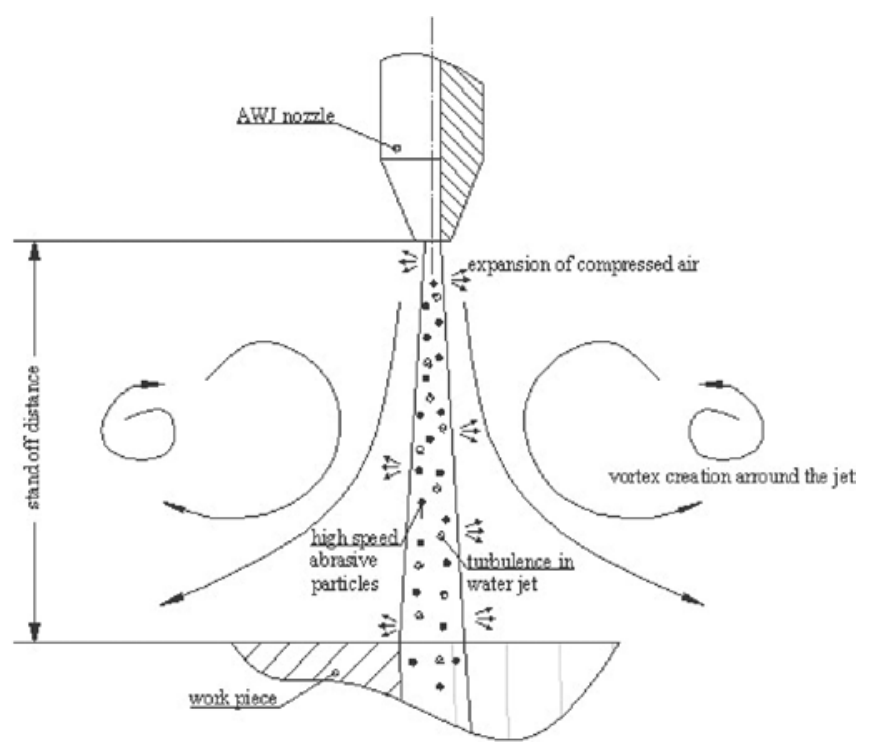

Fig. 7. Possible sound generation mechanisms in the stand-off distance area

distance area is shown on Fig. 7. In order to do that, a wide set of experiments with the minimising of all sound sources except one in the case of all five should be done. The synergy of this type of model with other models, like a material removal, surface generation, etc. could lead us to a full-scale AWJ process modelling.

The knowledge collected in this research can be used for many applications in the field of AWJ machining process control and also for other processes, where we deal with significant sound generation.

\section{Conclusions}

The main objective in the present work is to develop a methodology and create a system for the stand-off control in abrasive water jet (AWJ) machining processes. We found an evident connection between the stand-off distance and the sound generated during the straight-cut operation in the AWJ machining process. Some results of the presented research were successfully applied for an adaptive- control constraint AWJ system. For an adaptivecontrol system (ACS) of the stand-off distance, more tests have to be done. An adaptive on-line stand-off control system will enable the integration of the AWJ process in the automated production line. The Production will become more efficient by means of quality and costs.

Acknowledgement The work presented in the paper was supported by the bilateral project P. A. I. Proteus No. 99009, entitled "Intégration de procèdès non conventionnels de mise en forme en conception", between the Republic of France and the Republic of Slovenia.

\section{References}

1. Guo NS, Louis H, Meier G, et al. (1994) Abrasive water jet Cuttingmethods to calculate cutting performance and cutting efficiency. In: Rakowski Z (ed) Geomechanics 93. Balkema, Rotterdam, pp 291-299

2. Faber K, Oweinah H. (1991) Influence of process parameters on blasting performance with the abrasive-jet, jet cutting technology. In: Saunders D (ed) Proceedings of 10th international symposium. Amsterdam, The Netherlands, 31 October-2 November 1991. Elsevier, London, pp 365382

3. Gun NS (1994) Schneidprozess und Schnittqualitat beim Waserabrasivstrahlschneiden. VDI-fortschritt-berichte, Reihte 2, nr. 328

4. Machida T, Saeki K (1995) Potentiality of water jet method for cutting of sheet materials. In: Proceedings of the 8th American water jet technology conference. Houston, Texas, 26-29 August 1995, vol 1, pp 343-358

5. Chung Y, Geskin ES, Singh P (1992) Prediction of the geometry of the kerf created in the course of abrasive waterjet machining of ductile materials. In: Lichtarocich A (ed) Jet cutting technology. Kluwer, Dordrecht, pp 525-541

6. Kovacevic R, Wang L, Zhang YM (1993) Detection of AWJ nozzle wear using acoustic signature analysis. In: Proceedings of the 7th American water jet technology conference. Seattle, Washington, 28-31 August, 1993, vol 1, pp 217-231

7. Kovacevic R, Wang L, Zhang YM (1994) Identification of abrasive waterjet nozzle wear based on parametric spectrum estimation of acoustic signal. Proc Inst Mech Eng J Eng Manage 208:217-231 